\title{
Mammalian meiotic silencing exhibits sexually dimorphic features
}

\author{
J. M. Cloutier ${ }^{1}$ S. K. Mahadevaiah ${ }^{1} \cdot$ E. ElInati ${ }^{1}$ - A. Tóth $^{2} \cdot$ James Turner $^{1}$
}

Received: 16 July 2015 /Revised: 24 November 2015 / Accepted: 10 December 2015 / Published online: 28 December 2015

(C) The Author(s) 2015. This article is published with open access at Springerlink.com

\begin{abstract}
During mammalian meiotic prophase I, surveillance mechanisms exist to ensure that germ cells with defective synapsis or recombination are eliminated, thereby preventing the generation of aneuploid gametes and embryos. Meiosis in females is more error-prone than in males, and this is in part because the prophase I surveillance mechanisms are less efficient in females. A mechanistic understanding of this sexual dimorphism is currently lacking. In both sexes, asynapsed chromosomes are transcriptionally inactivated by ATR-dependent phosphorylation of histone H2AFX. This process, termed meiotic silencing, has been proposed to perform an important prophase I surveillance role. While the transcriptional effects of meiotic silencing at individual genes are well described in the male germ line, analogous studies in the female germ line have not been performed. Here we apply single- and multigene RNA fluorescence in situ hybridization (RNA FISH) to oocytes from chromosomally abnormal mouse models to uncover potential sex differences in the silencing response. Notably, we find that meiotic silencing in females is less efficient than in males. Within individual oocytes, genes located on the same asynapsed chromosome are silenced to differing extents, thereby generating mosaicism in gene expression profiles across oocyte populations. Analysis
\end{abstract}

This article is part of a Special Issue on "Recent advances in meiotic chromosome structure, recombination and segregation" edited by Marco Barchi, Paula Cohen and Scott Keeney.

James Turner

James.Turner@crick.ac.uk

1 The Francis Crick Institute, Mill Hill Laboratory, The Ridgeway, Mill Hill, London NW7 1AA, UK

2 Institute of Physiological Chemistry, Technische Universität Dresden, Dresden 01307, Germany of sex-reversed XY female mice reveals that the sexual dimorphism in silencing is determined by gonadal sex rather than sex chromosome constitution. We propose that sex differences in meiotic silencing impact on the sexually dimorphic prophase I response to asynapsis.

Keywords Meiosis · Meiotic silencing · Oocytes · Epigenetics $\cdot$ Checkpoints $\cdot$ Sex differences

\section{Introduction}

Meiosis is a dual cell division that halves the chromosome content of diploid germ cells. Defects in meiosis can result in gametes carrying the wrong chromosome number, and therefore the key chromosomal events that precede the meiotic divisions are monitored by surveillance pathways or checkpoints (Burgoyne et al. 2009; Handel and Schimenti 2010; Nagaoka et al. 2012). In mammals, these function at two stages. The first, the prophase I checkpoint, monitors homologous synapsis and recombination, while the second, the spindle assembly checkpoint (SAC), functions later at the metaphase/anaphase I transition and monitors bipolar attachment to the meiotic spindle (Burgoyne et al. 2009; Handel and Schimenti 2010; Nagaoka et al. 2012).

In mammals, most cases of human aneuploidy arise from maternal meiotic errors (Hunt and Hassold 2002; Morelli and Cohen 2005; Nagaoka et al. 2012). A number of distinct aetiological factors contribute to this sex bias. For example, in females, univalent chromosomes can readily form bipolar attachments at the first meiotic division, and in doing so, satisfy the requirements of the SAC (Kouznetsova et al. 2007). It is clear that the SAC is also weaker in females than in males. In males (XY), a univalent $\mathrm{X}$ chromosome triggers a robust $\mathrm{SAC}$ response, resulting in arrest at metaphase I (Burgoyne et 
al. 1992). However, in females with $X$ chromosome monosomy (XO females), oocytes can progress through the meiotic divisions despite the presence of a misaligned univalent $\mathrm{X}$ chromosome (LeMaire-Adkins et al. 1997). The increased efficiency of the SAC in males is at least in part due to a potentiating effect of the $\mathrm{Y}$ chromosome gene $Z f y 2$ (Vernet et al. 2011).

In contrast to events at metaphase I, sex differences in prophase I checkpoint control are less well studied. In males, problems in synapsis and/or recombination, arising either through chromosome abnormalities or targeted meiotic mutations, have variable effects on prophase I progression, ranging from normal germ cell development (Manterola et al. 2009) to complete pachytene loss (Burgoyne et al. 2009). In models where germ cell loss is observed, the effects are generally more severe in males than in females (Hunt and Hassold 2002; Kolas et al. 2004). This may be because in males, such defects disrupt Meiotic Sex Chromosome Inactivation (MSCI), the silencing of the $\mathrm{X}$ and $\mathrm{Y}$ chromosomes during prophase I (Mahadevaiah et al. 2008; McKee and Handel 1993). MSCI failure leads to misexpression of toxic sex-linked genes and subsequent midpachytene arrest (Royo et al. 2010). However, whether the prophase I checkpoint, like the SAC, is less robust in females than in males is unclear.

MSCI is a manifestation of a general mechanism, meiotic silencing, which acts in both sexes to inactivate genes on asynapsed chromosomes (Baarends et al. 2005; Turner et al. 2005). Although its purpose is unknown, meiotic silencing may serve a prophase I checkpoint function by starving germ cells of multiple essential gene products (Burgoyne et al. 2009). In view of its potential checkpoint function, there is a clear motivation for comparing meiotic silencing between males and females. Many components of the meiotic silencing pathway, including BRCA1 and $\gamma \mathrm{H} 2 \mathrm{AFX}$, are observed on asynapsed chromosomes in both sexes (Baarends et al. 2005; Garcia-Cruz et al. 2009; Kouznetsova et al. 2009; Turner et al. 2005; Wojtasz et al. 2009). However, a recent study noted that the male meiotic silencing mark lysine-9 trimethylated histone H3 (H3K9me3) was absent on asynapsed chromosomes in the female (Taketo and Naumova 2013). Whether this and other epigenetic dissimilarities create sex differences in gene expression from asynapsed chromosomes is not known. Such an analysis requires single cell transcriptional approaches, in which expression at a given gene can be correlated with the synaptic status of the chromosome on which it resides. Gene-specific fluorescence in situ hybridization (RNA FISH) is especially useful for this purpose but has not yet been applied to mouse prophase I oocytes. We therefore used this technique to characterise meiotic silencing in the female and to compare it with that in the male.

\section{Results}

\section{Asynapsed chromosomes exhibit sexually dimorphic epigenetic features}

Prior to our transcriptional studies, we wished to examine epigenetic differences in asynapsed chromosome between males and females. The localization of many components involved in meiotic silencing in males has been examined in oocytes, including SYCP3, HORMAD1/2, BRCA1, ATR and $\gamma$ H2AFX (Baarends et al. 2005; Fukuda et al. 2010; Garcia-Cruz et al. 2009; Kouznetsova et al. 2009; Shin et al. 2010; Turner et al. 2005; Wojtasz et al. 2009). SYCP3 and HORMAD1/2 together act to recruit BRCA1 and ATR to asynapsed chromosome axes, after which ATR translocates through axis-associated loops, causing gene silencing through the creation of $\gamma \mathrm{H} 2 \mathrm{AFX}$ (Daniel et al. 2011; Kouznetsova et al. 2009; Royo et al. 2013; Turner et al. 2004; Wojtasz et al. 2012). We examined a further two silencing components, MDC1 and SUMO1, which also act as loop-associated silencing effectors (Ichijima et al. 2011; Rogers et al. 2004; Vigodner and Morris 2005), as well as H3K9me3, the latter of which has been found to be absent from asynapsed chromosomes in oocytes (Taketo and Naumova 2013). We used $\mathrm{XO}$ mice as our female model system, in order to directly compare the asynapsed $\mathrm{X}$ chromosome in oocytes with that in wild type (XY) spermatocytes. The asynapsed X chromosome in each sex was identified using an antibody to HORMAD2 (Wojtasz et al. 2009). At least 50 oocytes and spermatocytes were studied for each meiotic silencing factor assayed.

We found that MDC1 and SUMO1 localised to the asynapsed $\mathrm{X}$ chromosome in $\mathrm{XO}$ females, as in $\mathrm{XY}$ males, during both pachynema and diplonema (Fig. 1a, b, 1a-d). In contrast, consistent with a previous study (Taketo and Naumova 2013), H3K9me3 patterns differed between the sexes (Fig. 1e, f). During pachynema, H3K9me3 was present both at centromeric heterochromatin and the asynapsed $\mathrm{X}$ chromosome in XY males. Enrichment of H3K9me3 at the asynapsed $\mathrm{X}$ chromosome was most clear during diplonema (Fig. 1e). However, during diplonema in XO females, H3K9me3 was enriched at centromeric heterochromatin but not on the asynapsed $\mathrm{X}$ chromosome (Fig. 1f). This sex difference in H3K9me3 was confirmed quantitatively (Fig. 1g, h). We conclude that the chromatin of asynapsed chromosomes exhibits sexually dimorphic epigenetic features.

\section{Meiotic silencing of genes on the asynapsed $\mathrm{X}$ chromosome is less efficient in females than in males}

We next used gene-specific RNA FISH to assay meiotic silencing of the asynapsed $\mathrm{X}$ chromosome in $\mathrm{XO}$ oocytes. We 
Fig. 1 Analysis of epigenetic differences in meiotic silencing in oocytes compared to spermatocytes. a XY pachytene spermatocyte stained for SYCP3 (magenta), HORMAD2 (green) and MDC1 (red), showing MDC1 accumulation in the sex chromatin (arrow). b XO pachytene oocyte stained for SYCP3 (magenta), HORMAD2 (green) and MDC1 (red), also showing MDC1 enrichment on the asynapsed $\mathrm{X}$ chromosome (arrow). c XY pachytene spermatocyte stained for SYCP3 (magenta), HORMAD2 (green) and SUMO-1 (red), showing accumulation of SUMO-1 in the sex chromatin (arrow). d XO pachytene oocyte stained for SYCP3 (magenta), HORMAD2 (green) and SUMO-1 (red), also showing SUMO-1 enrichment on the asynapsed $\mathrm{X}$ chromosome (arrow). e XY diplotene spermatocyte stained with SYCP3 to mark chromosome axes (magenta), HORMAD2 (green) to label the asynapsed $\mathrm{X}$ and Y chromosomes (arrow) and H3K9me3 (red), which shows enrichment in the chromatin of the $\mathrm{X}$ and $\mathrm{Y}$ chromosomes and also at constitutive heterochromatin (asterisks). A line (yellow) was drawn through the asynapsed X (arrow) to quantify HORMAD2 and H3K9me3 intensities (g). f XO diplotene oocyte showing no enrichment of $\mathrm{H} 3 \mathrm{~K} 9 \mathrm{me} 3$ in the chromatin of the asynapsed $\mathrm{X}$ chromosome (arrow). H3K9me3 staining is restricted to sites of constitutive heterochromatin (asterisks). A line (yellow) was drawn through the asynapsed $\mathrm{X}$ (arrow) to quantify HORMAD2 and $\mathrm{H} 3 \mathrm{~K} 9 \mathrm{me} 3$ intensities (h). $\mathbf{g}, \mathbf{h}$ Intensities of $\mathrm{H} 3 \mathrm{~K} 9 \mathrm{me} 3$ and HORMAD2 immunostaining quantified by densitometry across the indicated paths along (g) the asynapsed $\mathrm{X}$ in the spermatocyte from panel e, and along (h) the asynapsed $\mathrm{X}$ in diplotene $\mathrm{XO}$ oocyte from panel $\mathbf{f}$. Scale bars $=10 \mu \mathrm{m}$
A

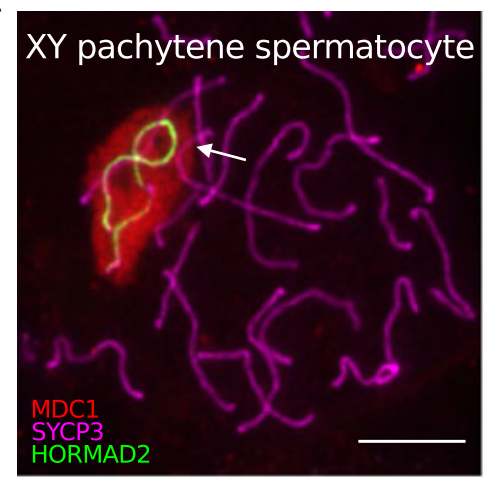

B

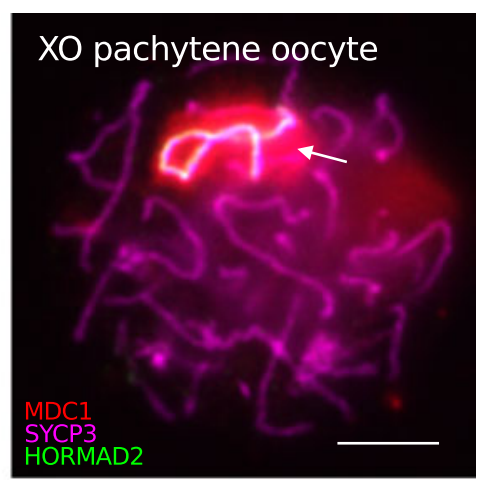

C

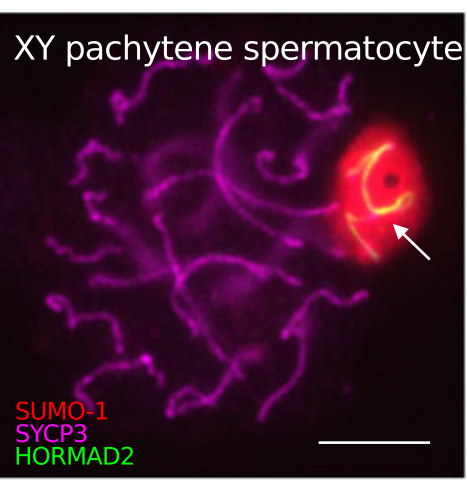

D

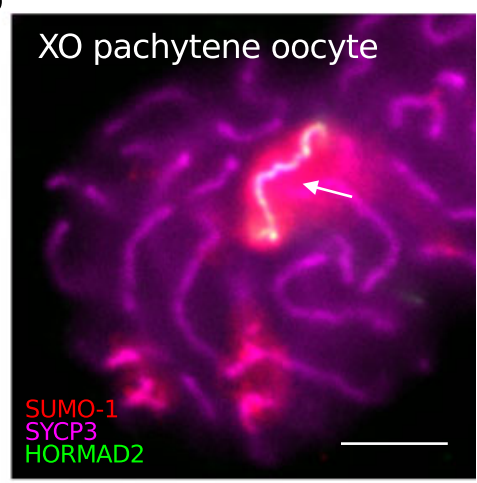

E

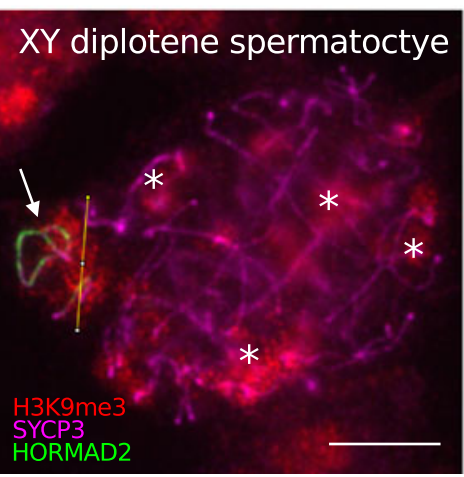

$\mathbf{F}$

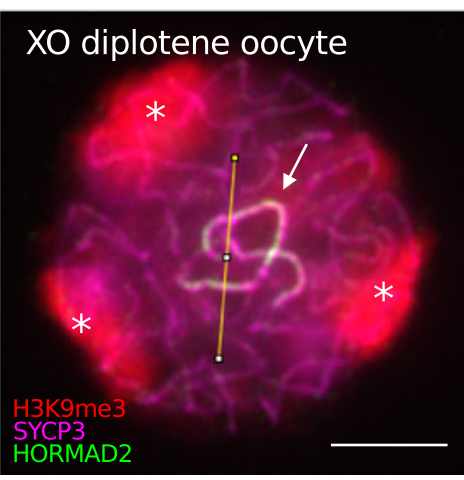

G

XY diplotene spermatocyte

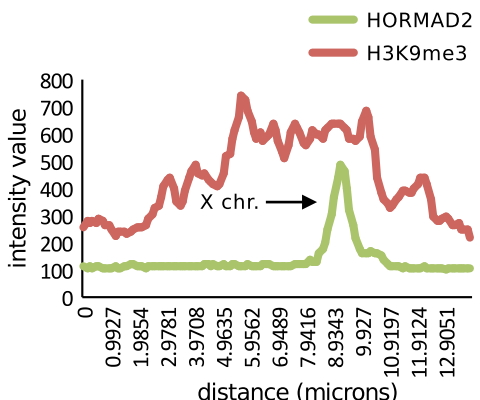

XO diplotene oocyte

HORMAD2 H3K9me3

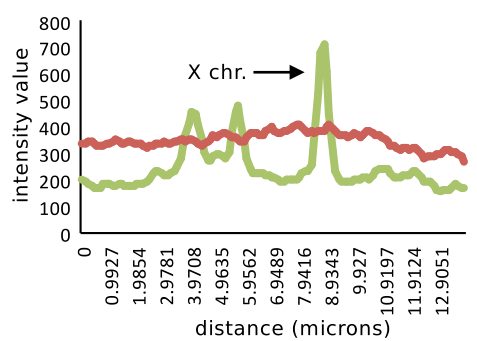

performed RNA FISH for three X-linked genes, $U t x, Z f x$ and $S \mathrm{cml}$. These genes are distant from each other on the X chromosome (Fig. 2a), and one, $Z f x$, has been shown to be essential for female fertility (Luoh et al. 1997). For each of the three genes, we carried out RNA FISH at four developmental time-points: $17.5,18.5,19.5$ and 20.5 days post coitum 
A
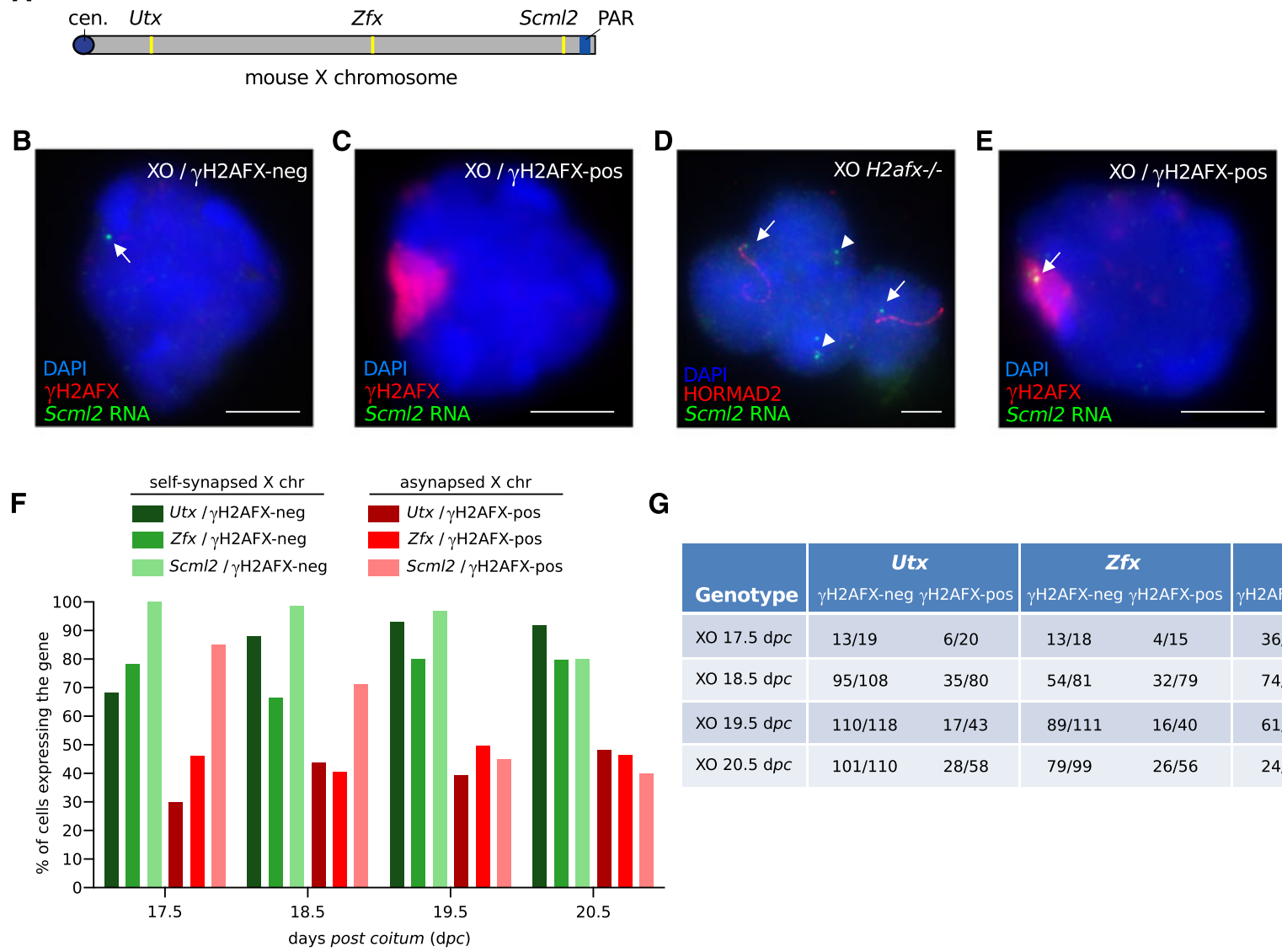

G

\begin{tabular}{|c|c|c|c|c|c|c|}
\hline \multirow{2}{*}{$\begin{array}{l}\text { Genotype } \\
\text { XO } 17.5 \mathrm{dpc}\end{array}$} & \multicolumn{2}{|c|}{$\begin{array}{c}\text { Utx } \\
\gamma \mathrm{H} 2 \mathrm{AFX} \text {-neg } \gamma \mathrm{H} 2 \mathrm{AFX} \text {-pos }\end{array}$} & \multicolumn{2}{|c|}{$\begin{array}{c}\text { ZfX } \\
\gamma H \text { H2AFX-neg } \gamma H \text { HAFX-pos }\end{array}$} & \multicolumn{2}{|c|}{$\begin{array}{c}\mathbf{S c m} / \mathbf{2} \\
\gamma \mathrm{H} 2 \mathrm{AFX} \text {-neg } \gamma \mathrm{H} 2 \mathrm{AFX} \text {-pos }\end{array}$} \\
\hline & $13 / 19$ & $6 / 20$ & $13 / 18$ & $4 / 15$ & $36 / 36$ & $69 / 81$ \\
\hline XO $18.5 \mathrm{dpc}$ & $95 / 108$ & $35 / 80$ & $54 / 81$ & $32 / 79$ & $74 / 75$ & $45 / 63$ \\
\hline XO $19.5 \mathrm{dpc}$ & $110 / 118$ & $17 / 43$ & $89 / 111$ & $16 / 40$ & $61 / 63$ & $14 / 31$ \\
\hline XO $20.5 d p c$ & $101 / 110$ & $28 / 58$ & $79 / 99$ & $26 / 56$ & $24 / 30$ & $8 / 20$ \\
\hline
\end{tabular}

H

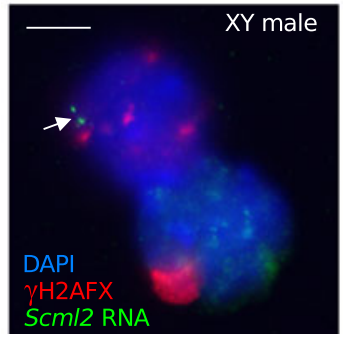

Fig. 2 Incomplete silencing of the asynapsed $\mathrm{X}$ chromosome in $\mathrm{XO}$ oocytes. a Schematic of mouse $\mathrm{X}$ chromosome showing the location of three genes, $U t x, Z f x$ and $S c m l 2$, which were used as RNA FISH probes to assess $\mathrm{X}$ chromosome transcription. $\mathrm{PAR}=$ pseudoautosomal region; cen. $=$ centromere. $\mathbf{b}$ Control XO oocyte with a self-synapsed $\mathrm{X}$ chromosome $(\gamma \mathrm{H} 2 \mathrm{AFX}$ domain-negative) and an RNA FISH signal (arrow; green), indicating expression of the X-linked gene Scml2. Oocytes were distinguished from somatic cells based upon DAPI staining and nuclear morphology. $\mathbf{c}$ XO oocyte with an asynapsed X chromosome ( $\gamma \mathrm{H} 2 \mathrm{AFX}$ domain-positive; red) and no RNA FISH signal, demonstrating silencing of $S c m l 2$. d Four adjacent XO $H 2 a f x-1-$ oocytes. The two middle nuclei have a self-synapsed X chromosome (HORMAD2-negative) and express Scml2 (arrowheads). The two outside nuclei have an asynapsed X chromosome (HORMAD2-positive; red) and also express $\mathrm{Scml} 2$ (arrows). Expression of Scml2 was observed in all XO H2afx-1oocytes with an asynapsed X chromosome $(n=34)$. e XO oocyte with

$(\mathrm{d} p c)$. This allowed us to track $\mathrm{X}$ chromosome silencing all the way through pachynema and diplonema: at $17.5 \mathrm{~d} p c$, most an asynapsed $\mathrm{X}$ chromosome ( $\gamma \mathrm{H} 2 \mathrm{AFX}$ domain-positive) with an RNA FISH signal (arrow), demonstrating expression of $S \mathrm{cml} 2$. f The percentage of XO oocytes expressing $U t x, Z f x$ and $S m c l 2$ at 17.5, 18.5, 19.5 and $20.5 \mathrm{~d} p c$. XO oocytes were subdivided into those without a $\gamma \mathrm{H} 2 \mathrm{AFX}$ domain, i.e. with a self-synapsed X chromosome (green bars), and those with a $\gamma \mathrm{H} 2 \mathrm{AFX}$ domain i.e. with an asynapsed $\mathrm{X}$ chromosome (red bars). One ovary was analysed for each gene and time point. g Raw data showing number of XO oocytes expressing $U t x$, $Z f x$ and $S c m l 2$ at 17.5, 18.5, 19.5 and $20.5 \mathrm{~d} p c$ out of the total number of oocytes analysed. $\mathbf{h}$ Robust silencing of X-genes in mid-late pachytene spermatocytes. Upper left nucleus: control zygotene spermatocyte with Scml2 RNA FISH signal. Lower right nucleus: mid-late pachytene spermatocyte with $\gamma \mathrm{H} 2 \mathrm{AFX}$-labelled sex body and no Scml2 RNA FISH signal, indicating meiotic silencing. i Raw data showing counts of mid-late pachytene spermatocytes with and without RNA FISH signals for each probe. Scale bars $=5 \mu \mathrm{m}$

oocytes are in pachynema, and by $19.5 \mathrm{~d} p c$, most oocytes are in late diplonema (Cloutier et al. 2015). 
In approximately one half of oocytes from XO females, the single $\mathrm{X}$ chromosome self-synapses, and as a result, does not accumulate $\gamma \mathrm{H} 2 \mathrm{AFX}$ labelling (Turner et al. 2005). Cot-1 and RNA polII analyses have previously shown that in these oocytes, the $\mathrm{X}$ chromosome escapes meiotic silencing (Baarends et al. 2005; Turner et al. 2005). We therefore used XO oocytes with self-synapsed $\mathrm{X}$ chromosomes as internal, positive controls in our female RNA FISH experiments. Within this positive control oocyte population, all three X-genes were expressed at the expected high frequency, with the majority of oocytes showing an RNA FISH signal (Fig. 2b, green bars in Fig. 2f, quantitation in Fig. 2g). This high frequency of expression for each X-gene was observed at all four timepoints analysed (green bars in Fig. 2f, quantitation in Fig. 2g).

Next, we assayed X-gene expression in XO oocytes with an asynapsed, $\gamma \mathrm{H} 2 \mathrm{AFX}$-positive $\mathrm{X}$ chromosome. For all three genes, we found that the percentage of expressing oocytes was lower than that observed in XO oocytes with a self-synapsed $\mathrm{X}$ chromosome, thereby confirming the presence of meiotic silencing at all three loci (Fig. 2c, red bars in Fig. 2f, quantitation in Fig. 2g). In order to confirm that this meiotic silencing was dependent on H2AFX, we then performed RNA FISH for one of the X-genes, Scml2, on XO H2afx - - - females at 19.5dpc (Celeste et al. 2002). All XO H2afx-/- oocytes with an asynapsed $\mathrm{X}$ chromosome, identified by immunostaining for the asynapsis marker HORMAD2, expressed Scml2 (34/ 34 , i.e. $100 \%$ oocytes expressing; Fig. 2 d). This frequency of expression was higher than that observed in XO H2afx +/+ oocytes with an asynapsed X chromosome (only 14/31, i.e. $45 \%$ oocytes expressing; Fig. $2 \mathrm{c}$, f and g) and was similar to that in XO H2afx +/+ oocytes with a self-synapsed X chromosome (61/63, i.e. $97 \%$ oocytes expressing; Fig. $2 b$, $f$ and g). Thus, meiotic silencing in oocytes is H2afx-dependent.

Although meiotic silencing was clearly operating in $\mathrm{XO}$ females, we were surprised to find sizeable populations of oocytes with an asynapsed $\mathrm{X}$ chromosome in which $U t x, Z f x$ and $S c m l 2$ RNA FISH signals were present, despite the coexistence of $\gamma \mathrm{H} 2 \mathrm{AFX}$ silencing domains (Fig. 2e, red bars in Fig. 2f, quantitation in Fig. $2 \mathrm{~g}$ ). For example, at $19.5 \mathrm{~d} p c, U t x$, $Z f x$ and $S c m l 2$ were expressed in 39, 40 and $45 \%$ of oocytes with an asynapsed $\mathrm{X}$ chromosome, respectively. This phenomenon was observed at all developmental time-points analysed (Fig. 2f, g). In order to ascertain whether this "leakiness" in meiotic silencing was specific to females, we then performed RNA FISH for $U t x, Z f x$ and $S c m l 2$ in wild type, XY males, focusing our analysis on mid-late pachytene spermatocytes. Silencing of each of the three X-genes in spermatocytes was highly efficient, with $U t x, Z f x$ and $S c m l 2$ RNA FISH signals present in 0,1 and $1 \%$ of spermatocytes, respectively (Fig. $2 \mathrm{~h}, \mathrm{i}$ ). We conclude that meiotic silencing is less efficient in oocytes than in spermatocytes.

\section{Inefficient meiotic silencing in oocytes also affects asynapsed autosomes}

XO females exhibit perinatal germ cell losses (Burgoyne and Baker 1985) that preferentially affect oocytes with asynapsed $X$ chromosomes (Cloutier et al. 2015). We considered the possibility that silencing in some $\mathrm{XO}$ oocytes might be highly efficient, and that these oocytes were preferentially eliminated, and thus missing from our RNA FISH analysis. This was unlikely, because our XO experiments included oocytes harvested at $17.5 \mathrm{~d} p c$ (Fig. 2f, g) when germ cell elimination has not yet initiated in XO females (Burgoyne and Baker 1985). Nevertheless, to further exclude an effect of selection on our RNA FISH results, we performed RNA FISH on another model, in which the presence of an asynapsed chromosome does not elicit prophase I elimination. Tc1 females (O'Doherty et al. 2005) carry a copy of human chromosome 21 (h21), which self-synapses in $60 \%$ of pachytene oocytes and is asynapsed in the remaining oocytes. Importantly, Tc1 oocytes with an asynapsed h21 chromosome persist from pachynema through diplonema (Cloutier et al. 2015).

We performed RNA FISH on Tc1 oocytes for three genes, USP25, NRIP1 and TPTE, which are located at different sites on the h21 chromosome (Fig. 3a). As with our XO experiments, we carried out RNA FISH for each of the three genes at four developmental time-points: 17.5, 18.5, 19.5 and 20.5 $\mathrm{d} p c$. Tc1 oocytes with a self-synapsed $\mathrm{h} 21$ chromosome, which acted as RNA FISH positive controls, exhibited high frequencies of expression for each of the three genes, at all gestational ages (Fig. 3b, green bars in Fig. 3e, quantitation in Fig. 3f). Those with an asynapsed h21 chromosome exhibited lower expression frequencies, consistent with the presence of meiotic silencing (Fig. 3c, red bars in Fig. 3e, quantitation in Fig. 3f). However, reminiscent of our observations in XO females, in $\mathrm{Tc} 1$ females, many oocytes with asynapsed $\mathrm{h} 21$ chromosomes exhibited RNA FISH signals for the three genes studied. For example, at $19.5 \mathrm{~d} p c, U S P 25, N R I P 1$ and TPTE were expressed in 30, 30 and $65 \%$ of oocytes with an asynapsed Tc1 chromosome, respectively (Fig. 3e, f). We then analysed the expression of the same genes in Tc1 males. USP25 and TPTE were expressed in spermatogenic cells, while expression of NRIP1 could not be detected. Importantly, in Tc1 pachytene cells with an asynapsed Tc1 chromosome, silencing of both USP25 and TPTE was efficient (Fig. 3g, h). Thus, escape from silencing is not a feature specific to the $\mathrm{XO}$ female mouse model, nor indeed to the $\mathrm{X}$ chromosome.

\section{Meiotic silencing creates mosaic gene expression patterns in oocytes}

We next questioned whether escape from meiotic silencing was concerted, affecting multiple genes on the same asynapsed chromosome simultaneously, or stochastic, 
affecting different genes independently of one another. To discriminate between these possibilities, we performed multicolour, triple RNA FISH for $U t x, Z f x$ and $S c m l 2$ in XO females at $19.5 \mathrm{~d} p c$. As expected, the majority of XO oocytes with a self-synapsed X chromosome exhibited RNA FISH signals for all three genes simultaneously (Fig. 4a; quantitation in Fig. 4c). Interestingly, however, in XO oocytes with an asynapsed $\mathrm{X}$ chromosome, different combinations of gene expression were observed. Twelve percent $(n=51)$ of oocytes with an asynapsed $\mathrm{X}$ chromosome expressed $U t x, Z f x$ and Scml2 simultaneously (Fig. 4c). However, within the remaining $88 \%$ of oocytes, escape from silencing could be observed at none, at one, or at two of the X-genes studied, in roughly equal proportions. Thus, silencing across the $\mathrm{X}$ chromosome is stochastic. We observed the same phenomenon of stochastic gene silencing in Tc1 females using simultaneous triple RNA FISH for USP25, NRIP1 and TPTE at $19.5 \mathrm{~d} p c$ (Fig. 4d-f). Thus, meiotic silencing creates mosaicism in gene expression patterns between oocytes.

\section{Gonadal sex determines male female differences in meiotic silencing}

Sexual dimorphisms can be controlled by male/female differences in sex chromosome genotype, e.g. the dose of $\mathrm{X}$ chromosomes, or the presence or absence of a $\mathrm{Y}$ chromosome. Alternatively, they can be regulated by the gonadal environment, i.e. the presence of a testis or an ovary (Arnold et al. 2012). We wished to establish whether sex chromosome complement or gonadal sex governed the sex difference in meiotic silencing efficiency that we had identified. Our data excluded an effect of $\mathrm{X}$ chromosome dose, because sex differences in meiotic silencing were observed in $\mathrm{XO}$ females and $\mathrm{XY}$ males, despite them both carrying a single $\mathrm{X}$ chromosome. However, a possible enhancing effect of the $\mathrm{Y}$ chromosome on meiotic silencing in males had not been considered. We therefore performed RNA FISH for the X-linked gene $S \mathrm{~cm} / 2$ in sex-reversed $X^{\mathrm{d} 1}$ females (Mahadevaiah et al. 1998), in which oocytes contain an $\mathrm{X}$ and a $\mathrm{Y}$ chromosome. As in $\mathrm{XO}$ females, we observed escape of $S c m l 2$ from silencing in $\mathrm{XY}^{\mathrm{d} 1}$ pachytene oocytes $(81 / 143$, i.e. $57 \%$ oocytes expressing; Fig. 5a-d) at frequencies that far-exceeded those seen in XY males (Fig. 2h, i). Higher levels of escape from silencing in $X Y^{\mathrm{d} 1}$ oocytes compared to $\mathrm{XY}$ spermatocytes were also observed for $U t x$ and $Z f x$ (Fig. 5d). Thus, sex differences in meiotic silencing are driven by gonadal sex and not by sex chromosome constitution.

\section{Discussion}

Chromosome abnormalities confer greater germ cell loss in males than in females (Burgoyne et al. 2009). This is due in
Fig. 3 Incomplete silencing of the asynapsed h21 chromosome in Tc1 oocytes. a Schematic of Tc1 h21 chromosome showing the location of three genes, TPTE, NRIPI and USP25, which were used as RNA FISH probes to assess $\mathrm{h} 21$ chromosome transcription. cen. = centromere. $\mathbf{b}$ Control Tc1 oocyte with a self-synapsed $\mathrm{h} 21$ chromosome $(\gamma \mathrm{H} 2 \mathrm{AFX}$ domain-negative) and an RNA FISH signal (arrow; green), indicating expression of the X-linked gene USP25. $\mathbf{c}$ Tc1 oocyte with an asynapsed h21 chromosome ( $\gamma \mathrm{H} 2 \mathrm{AFX}$ domain-positive; red) and no RNA FISH signal, demonstrating silencing of USP25. d Tc1 oocyte with an asynapsed $\mathrm{h} 21$ chromosome ( $\gamma \mathrm{H} 2 \mathrm{AFX}$ domain-positive) and an RNA FISH signal (arrow), demonstrating expression of USP25. e The percentage of Tc1 oocytes expressing USP25, NRIP1 and TPTE at 17.5, 18.5, 19.5 and $20.5 \mathrm{~d} p c$. Tc1 oocytes were subdivided into those without a $\gamma \mathrm{H} 2 \mathrm{AFX}$ domain, i.e. with a self-synapsed Tc1 chromosome (green bars) and those with a $\gamma \mathrm{H} 2 \mathrm{AFX}$ domain, i.e. with an asynapsed $\mathrm{X}$ chromosome (red bars). One ovary was analysed for each gene and time point. $\mathbf{f}$ Raw data showing number of Tc1 oocytes expressing USP25, NRIP1 and TPTE at 17.5, 18.5, 19.5 and $20.5 \mathrm{~d} p c$ out of the total number of oocytes analysed. g Robust silencing of Tc1 genes in mid-late pachytene Tc1 spermatocytes. Upper left nucleus: spermatid with USP25 RNA FISH signal. Middle nucleus: mid-late pachytene spermatocyte with $\gamma \mathrm{H} 2 \mathrm{AFX}-$ labelled sex body and no USP25 RNA FISH signal, indicating meiotic silencing. $\mathbf{h}$ Raw data showing counts of mid-late pachytene spermatocytes with and without RNA FISH signals for USP25 and TPTE. NRIP1 is not expressed in Tc1 testes. Scale bar $=5 \mu \mathrm{m}$

part to the reduced stringency of the metaphase I spindle checkpoint in females (LeMaire-Adkins et al. 1997; Nagaoka et al. 2011) but is also thought to reflect illdefined sex differences in the efficacy of the prophase I response to asynapsis (Hunt and Hassold 2002; Morelli and Cohen 2005; Nagaoka et al. 2012). Here we shed light on this sex difference by demonstrating that meiotic silencing in the female germ line is less efficient than in the male. Although the role of meiotic silencing in mammalian infertility is unclear, it may trigger prophase I elimination by rendering germ cells deficient in multiple gene products. Under this model, the detrimental effects of meiotic silencing would increase as a function of its efficiency, thereby providing an explanation as to why chromosome abnormalities cause more severe germ cell loss in males than in females.

Our multigene RNA FISH analysis shows that genes located on the same asynapsed chromosome are silenced to varying extents. In addition, the combination of genes that are silenced on a given asynapsed chromosome differs between oocytes. A potential caveat of this observation is that the efficiency of probe hybridization may be reduced when used in a multiplexing experiment. However, the mosaicism, assayed here at the level of nascent RNA, could create distinct gene expression profiles that disturb different biological pathways, both qualitatively and quantitatively. Thus, in XO females, and other chromosomally abnormal mouse models exhibiting prophase I germ cell losses, the precise cause of arrest could differ from oocyte to oocyte depending on the suite of genes that are silenced. 
A
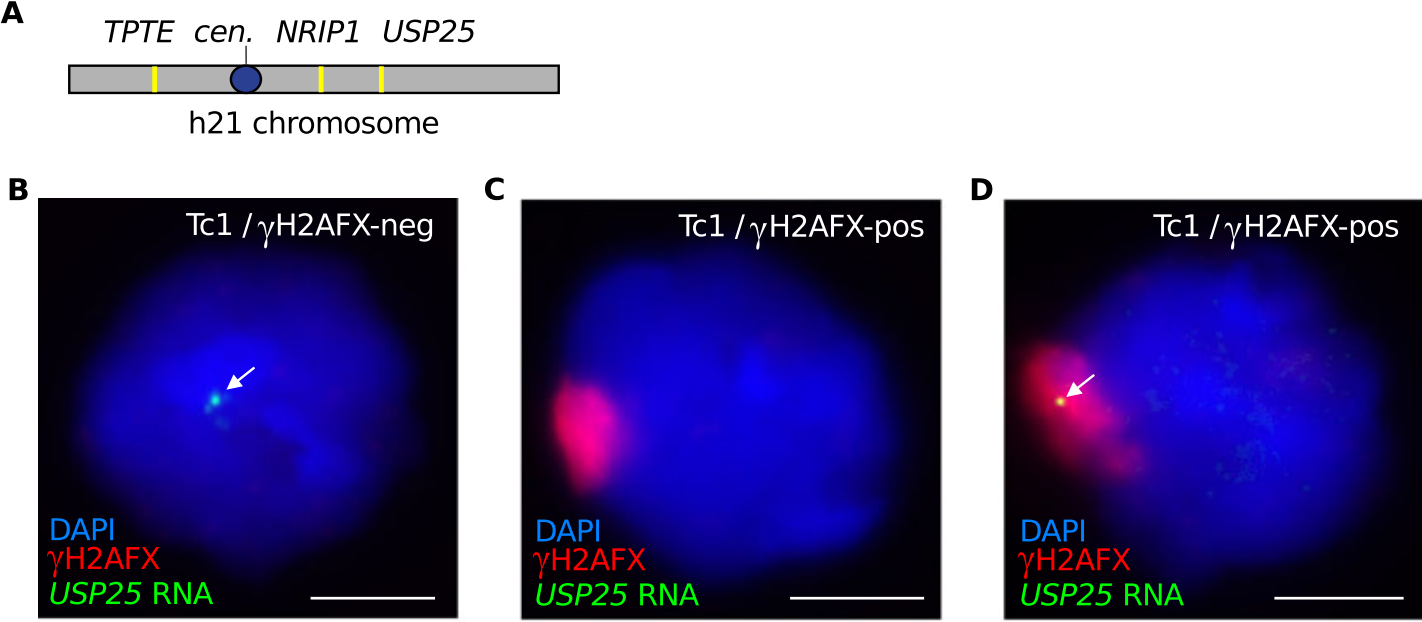

E
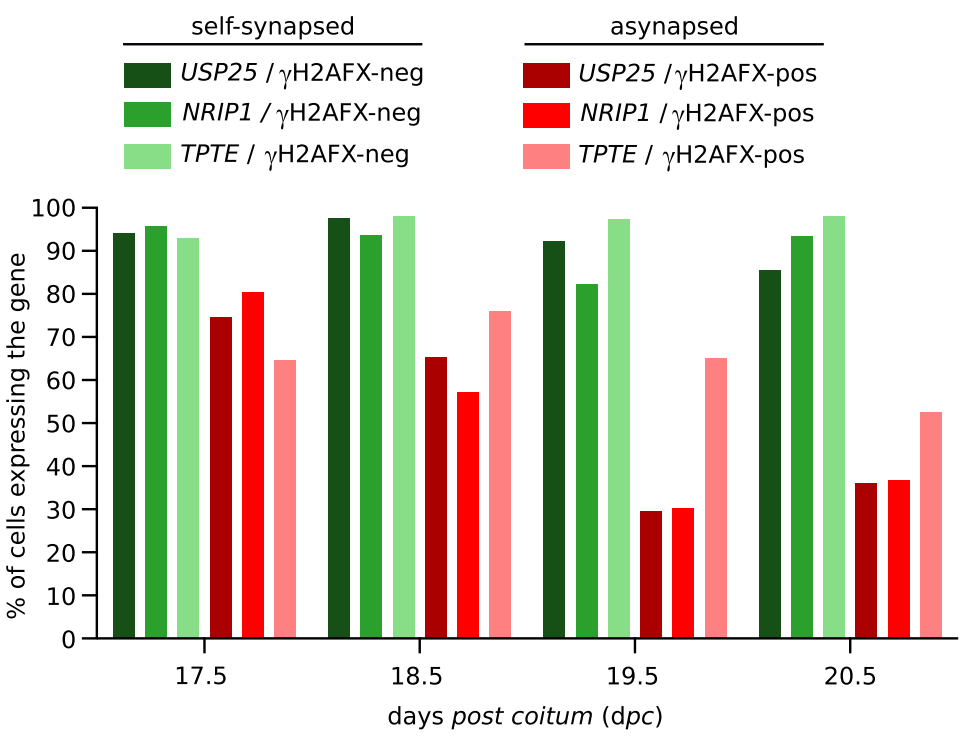

F

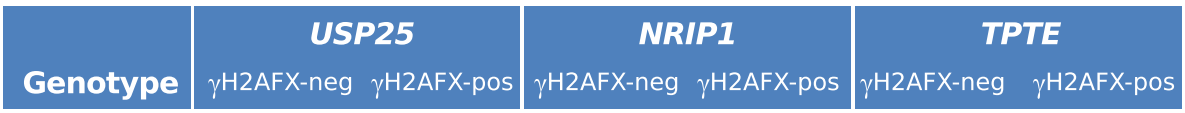

\begin{tabular}{|c|c|c|c|c|c|c|}
\hline Tc1 $17.5 \mathrm{~d} p c$ & $79 / 84$ & $50 / 67$ & $68 / 71$ & $33 / 41$ & $13 / 14$ & $22 / 34$ \\
\hline Tc1 $18.5 \mathrm{dpc}$ & $120 / 123$ & $51 / 78$ & $59 / 63$ & $20 / 35$ & $104 / 106$ & $76 / 100$ \\
\hline Tc1 $19.5 \mathrm{dpc}$ & $84 / 91$ & $8 / 27$ & $152 / 182$ & $7 / 23$ & $177 / 182$ & $15 / 23$ \\
\hline Tc1 $20.5 \mathrm{dpc}$ & $89 / 104$ & $22 / 61$ & $142 / 152$ & $7 / 19$ & $149 / 152$ & $10 / 19$ \\
\hline
\end{tabular}

G

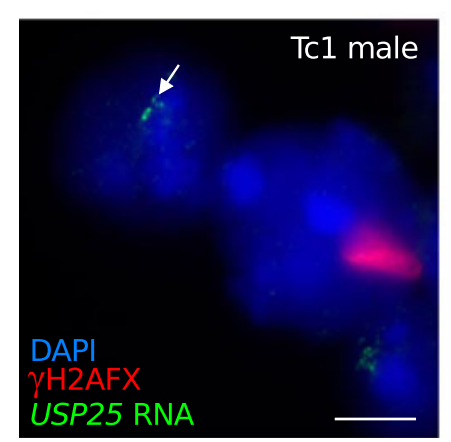

H

\begin{tabular}{|c|c|c|c|}
\hline Genes & $\begin{array}{c}\text { No. of mid/late pach } \\
\text { spermatocytes analyzed }\end{array}$ & $\begin{array}{c}\text { Spermatocytes without } \\
\text { RNA FISH signal }\end{array}$ & $\begin{array}{c}\text { Spermatocytes with } \\
\text { RNA FISH signal }\end{array}$ \\
\hline USP25 & 100 & 99 & 1 \\
\hline TPTE & 16 & 16 & 0 \\
\hline
\end{tabular}




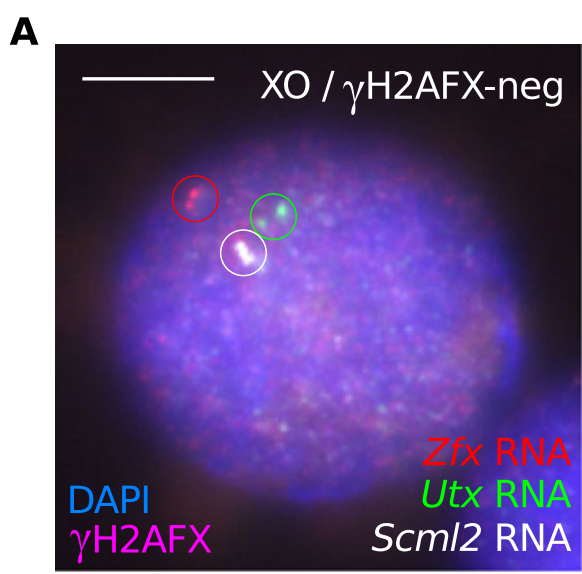

D

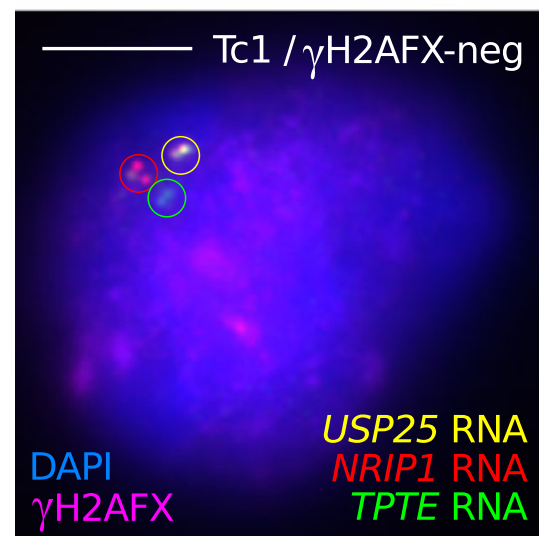

B

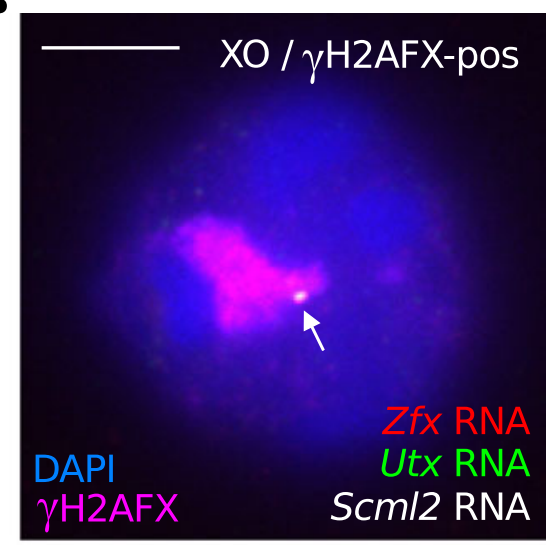

E

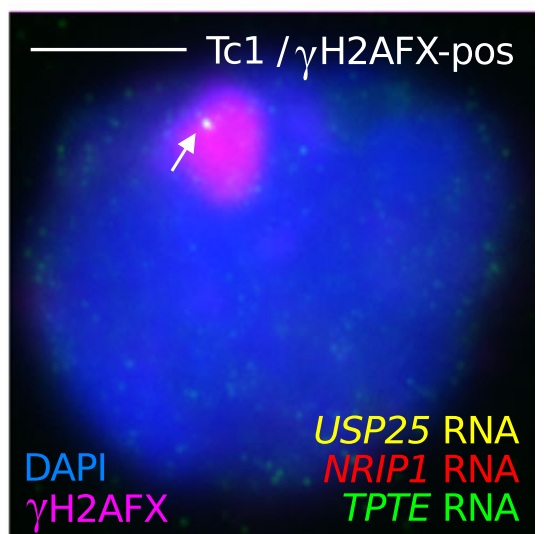

C

XO multigene RNA FISH

$\gamma \mathrm{H} 2 \mathrm{AFX}$-neg oocytes $(n=50)$

3 genes active $=60 \%$

yH2AFX-pos oocytes $(n=51)$
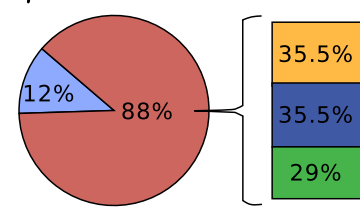
$\square \geq 1$ gene silent $\left\{\begin{array}{l}\square 1 \text { gene silent } \\ \square 2 \text { genes silent }\end{array}\right.$ $\square 3$ genes active $\square \square 3$ genes silent

$F$

Tc1 multigene RNA FISH

$\gamma \mathrm{H} 2 \mathrm{AFX}$-neg oocytes $(n=274)$

3 genes active $=89 \%$

$\gamma$ H2AFX-pos oocytes $(n=126)$

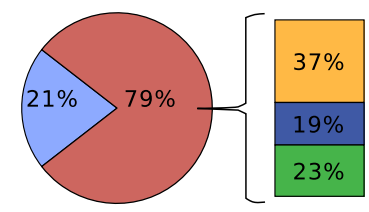

$\square \geq 1$ gene silent $\left\{\begin{array}{l}\square 1 \text { gene silent } \\ \square 2 \text { genes silent }\end{array}\right.$

$\square 3$ genes active $\square \square 3$ genes silent
Fig. 4 Mosaic silencing of asynapsed chromosomes in oocytes. a-c Simultaneous triple RNA FISH was performed in XO oocytes using probes for Utx, Zfx and $S c m l 2$ at $19.5 \mathrm{~d} p c$. a Control XO oocyte with a self-synapsed $\mathrm{X}$ chromosome ( $\gamma \mathrm{H} 2 \mathrm{AFX}$ domain-negative) and RNA FISH signals for all three genes (circled; red, green and white). b XO oocyte with an asynapsed X chromosome ( $\gamma \mathrm{H} 2 \mathrm{AFX}$ domain-positive; magenta) and an RNA FISH signal only for Scml2 (arrow), indicating that the silencing response is mosaic, inactivating two of the three genes analysed. c Quantitation of RNA FISH data. $60 \%$ of $\gamma \mathrm{H} 2 \mathrm{AFX}$-negative $\mathrm{XO}$ oocytes express all three genes simultaneously. The pie chart shows the percentage of $\mathrm{XO}$ oocytes with an asynapsed $\mathrm{X}$ chromosome that has at least one gene silenced (88\%). The accompanying bar chart shows the percentage of oocytes with one, two and three genes silenced. $n$ represents the number of oocytes analysed from one $19.5 \mathrm{~d} p c$ ovary. d-f Simultaneous triple RNA FISH was performed in Tc1 oocytes using the probes for USP25, NRIP1 and TPTE at 19.5dpc. d Control Tc1 oocyte with a self-synapsed $\mathrm{h} 21$ chromosome $(\gamma \mathrm{H} 2 \mathrm{AFX}$ domain-negative, inset $)$

Why should meiotic silencing be more robust in males than in females? During male meiosis, the asynapsed $\mathrm{X}$ and $\mathrm{Y}$ chromosomes are transcriptionally inactivated by MSCI. Defects in MSCI cause complete midpachytene arrest, due to the misexpression of toxic sex-linked genes, e.g. Zfyl and Zfy2 (Royo et al. 2010). We propose that meiotic silencing in males must be highly efficient in order to prevent the misexpression of these XY-encoded pachytene-lethal genes during normal male meiosis.

Although most components of the meiotic silencing pathway are conserved between the sexes, $\mathrm{H} 3 \mathrm{~K} 9 \mathrm{me} 3$ is present on and RNA FISH signals for all three genes (circled), showing active transcription at all three loci. e Tc1 oocyte with an asynapsed h21 chromosome ( $\gamma \mathrm{H} 2 \mathrm{AFX}$ domain-positive, inset $)$ and only an RNA FISH signal for USP25 (arrow), indicating that the silencing response is mosaic. f Quantitation of RNA FISH data. $89 \%$ of $\gamma \mathrm{H} 2 \mathrm{AFX}$-negative Tc1 oocytes express all three genes simultaneously. The pie chart shows the percentage of $\mathrm{Tc} 1$ oocytes with an asynapsed $\mathrm{h} 21$ that have at least one gene silenced (79\%). The accompanying bar chart shows the percentage of oocytes with one, two and three genes silenced. $n$ represents the number of oocytes analysed from two $19.5 \mathrm{~d} p c$ ovaries. Scale bars $=5 \mu \mathrm{m}$. Note that RNA FISH signals appear in some cells appear as double dots and in others as single dots. At this stage of germ cell development, each locus will be comprised of two sister chromatids. Double spots most likely represent expression from sisters that are spatially separate, while single spots represent expression from sisters that are in close proximity to each other

asynapsed chromosomes in the male but not in the female. Our data indicate that $\mathrm{H} 2 \mathrm{AFX}$ phosphorylation creates mosaicism in gene expression patterns, while additional, male-specific chromatin changes, including $\mathrm{H} 3 \mathrm{~K} 9 \mathrm{me} 3$, result in stable and complete silencing. Identification of the histone methyltransferases that catalyse $\mathrm{H} 3 \mathrm{~K} 9$ methylation on asynapsed chromosomes represents an important challenge in further understanding sex differences in the prophase I response to asynapsis. It will also be important to determine whether other factors involved in meiotic silencing in males (Becherel et al. 2013; Modzelewski et al. 2012) exhibit similar sexual dimorphisms. 
A

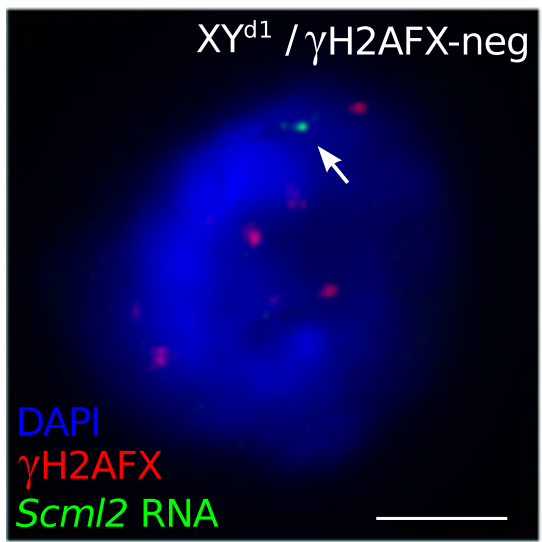

C

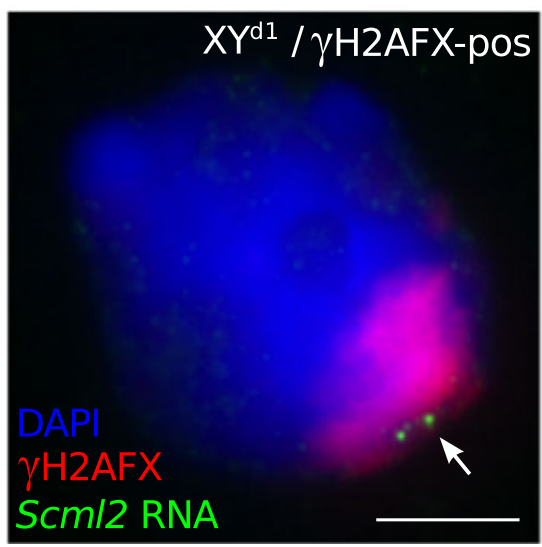

B

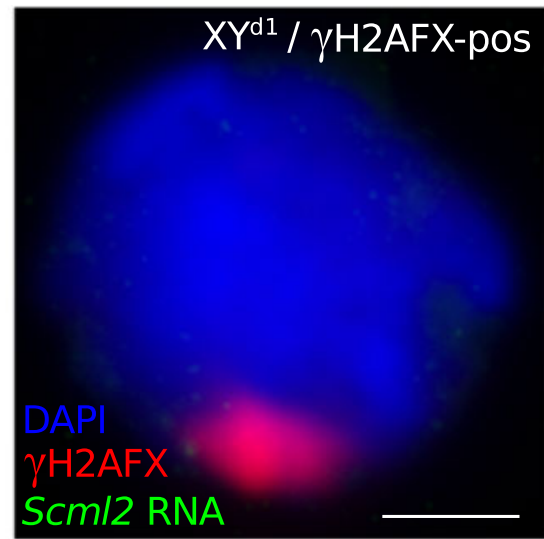

D

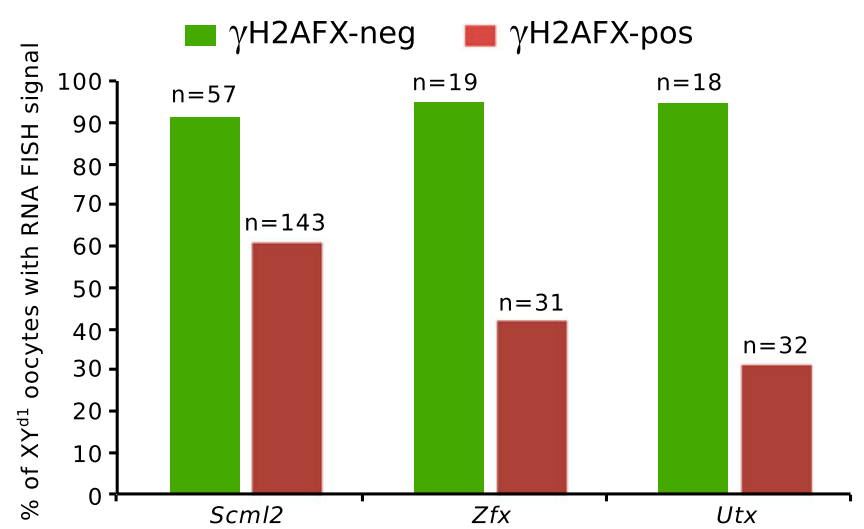

Fig. $5 \mathrm{X}$ chromosome silencing is inefficient in oocytes containing a $\mathrm{Y}$ chromosome. a Control XY ${ }^{\mathrm{d} 1}$ oocyte with no $\gamma \mathrm{H} 2 \mathrm{AFX}$ domain and an RNA FISH signal for $S c m l 2 . \mathbf{b} X Y^{\mathrm{d} 1}$ oocyte with a $\gamma \mathrm{H} 2 \mathrm{AFX}$ domain and no RNA FISH signal for $S c m l 2$, indicating silencing. $\mathbf{c} X Y^{\mathrm{d} 1}$ oocyte with a $\gamma \mathrm{H} 2 \mathrm{AFX}$ domain and a RNA FISH signal for $S \mathrm{cml} 2$, indicating escape

\section{Materials and methods}

\section{Animals}

Females were set up in matings and checked daily for copulation plugs. The day of plugging was considered 0.5 days post coitum $(\mathrm{d} p c)$. Embryos were sacrificed at 17.5, 18.5, 19.5 and $20.5 \mathrm{~d} p c$ using UK Home Office Schedule I methods. Ovaries were dissected from embryos and flash frozen in liquid nitrogen. Material was stored at $-80^{\circ} \mathrm{C}$ until later use. XO mice were generated on a random bred MF1 background (NIMR stock) by mating $\mathrm{XX}$ females to fertile $\mathrm{X}^{\mathrm{Y}^{*}} \mathrm{O}$ males, which harbour an $\mathrm{X}$ chromosome fused with a $\mathrm{Y}$ chromosome and give rise to "O" gametes (Eicher et al. 1991). H2afx-/- mice (Celeste et al. 2002) were generated on the MF1 background. XO $H 2 a f x-/-$ mice were generated by crossing $\mathrm{X}^{\mathrm{Y}} \mathrm{O} H 2 a f x+/-$ males with XX $H 2 a f x+/-$ females. Tc1 mice (O'Doherty et al. 2005) were maintained on the MF1 background. XY ${ }^{\mathrm{d} 1}$ females were produced on an MF1 from silencing. $\mathbf{d}$ Graph of the percentage of $X Y^{\mathrm{d} 1}$ oocytes with an RNA FISH signal for $S c m l 2, Z f x$ and $U t x . X^{\mathrm{d} 1}$ oocytes were subdivided into $\gamma \mathrm{H} 2 \mathrm{AFX}$ domain-negative (green bars) and $\gamma \mathrm{H} 2 \mathrm{AFX}$-positive oocytes (red bars). $n$ is the number of oocytes analysed from one $18.5 \mathrm{~d} p c$ ovary for each gene

background by mating $X Y$ males to sex-reversed $X Y^{d 1}$ females (Mahadevaiah et al. 1998).

\section{Chromosome spreads and RNA FISH}

Surface spreads were performed as previously described (Turner et al. 2004, 2005). Briefly, previously frozen $\left(-80{ }^{\circ} \mathrm{C}\right)$ ovaries were macerated in Roswell Park Memorial Institute (RPMI) on Superfrost slides, cells were permeabilized for $10 \mathrm{~min}$ in $0.05 \%$ Triton X-100 in distilled water and then fixed for $60 \mathrm{~min}$ in $2 \%$ formaldehyde, $0.02 \%$ SDS in phosphate-buffered saline (PBS). The slides were rinsed in distilled water, allowed to air dry and then blocked in PBT $(0.15 \%$ BSA, $0.10 \%$ TWEEN-20 in PBS) for 60 min. Slides were incubated with the following antibodies in a humid chamber overnight at $37{ }^{\circ} \mathrm{C}$ : rabbit anti-SYCP3 (1:100, Abcam: ab15093), mouse anti- $\gamma \mathrm{H} 2 \operatorname{AFX}(1: 100$, Upstate: 16-193), guinea pig and rabbit anti-HORMAD2 (1:200, ref. Wojtasz et al. 2009), rabbit anti-H3K9me3 (1:100, Upstate 
07-442), rabbit anti-SUMO1 (1:100, Abcam: ab32058) and sheep anti-MDC1 (1:10, Serotec: AHP799). Secondary antibodies (AlexaFluor 488, 594 and 647, Invitrogen) were applied 1:500 in PBS for 1 hour at $37^{\circ} \mathrm{C}$ and mounted in Vectashield with 4',6-diamidino-2-phenylindole (DAPI).

RNA FISH was carried out as previously described (Mahadevaiah et al. 2009). Briefly, previously frozen $\left(-80{ }^{\circ} \mathrm{C}\right)$ ovaries were mascerated in RPMI on Superfrost slides, cells were permeabilized for $10 \mathrm{~min}$ in chilled CSK buffer $(100 \mathrm{mM} \mathrm{NaCl}, 300 \mathrm{mM}$ sucrose, $3 \mathrm{mM} \mathrm{MgCl} 2$, $10 \mathrm{mM}$ PIPES, $0.5 \%$ Triton X-100, $1 \mathrm{mM}$ EGTA and $2 \mathrm{mM}$ vanadyl ribonucleoside, $\mathrm{pH}$ 6.8) and then fixed for $10 \mathrm{~min}$ in chilled $4 \%$ paraformaldehyde. Slides were then washed in PBS, dehydrated in a series of ethanol dilutions $(2 \times 70 \%, 80 \%, 95 \%, 100 \%)$ and air dried.

RNA FISH digoxigenin-labelled probes were prepared from $1 \mu \mathrm{g}$ of BAC DNA (from CHORI: Scml2, RP24204O18; Zfx, RP24-204018; USP25, RP11-296D11; NRIP1, RP11-22D1; from ABgene: TPTE, CTD-2260D15; Utx, gift from Mike Mitchell, University Marseilles) using the Biotin Nick Translation Kit (Roche), according to manufacturer's instructions. For each probe, $100 \mathrm{ng}$ digoxigeninlabelled BAC was prepared in $15 \mu \mathrm{l}$ formamide (Sigma), with $3 \mu \mathrm{g}$ mouse (for XO) or human (for Tc1) Cot1 DNA (Invitrogen) and $10 \mu \mathrm{g}$ sheared salmon sperm DNA (Ambion). Probes were denatured for $10 \mathrm{~min}$ at $80{ }^{\circ} \mathrm{C}$ and combined with $15 \mu \mathrm{l}$ pre-warmed $\left(37^{\circ} \mathrm{C}\right) 2 \times$ hybridization buffer ( $2 \times$ saline sodium citrate (SSC), $10 \%$ dextran sulphate (Sigma), $1 \mathrm{mg} / \mathrm{ml} \mathrm{BSA}$ and $2 \mathrm{mM}$ vanadyl ribonucleoside) and incubated for $30 \mathrm{~min}$ at $37^{\circ} \mathrm{C}$. Finally, $30-\mu$ l pre-hybridized probes were applied to slides and incubated in a humid chamber overnight at $37^{\circ} \mathrm{C}$.

The next day, slides were washed at $42{ }^{\circ} \mathrm{C}$, three times in $2 \times \mathrm{SSC}$ and $50 \%$ formamide, and three times in $2 \times \mathrm{SSC}$, for 5 min per wash. Slides were then transferred to $4 \times$ SSC and $0.1 \%$ TWEEN-20, and then blocked ( $4 \times \mathrm{SSC}, 4 \mathrm{mg} / \mathrm{ml}$ ) bovine serum albumin and $0.1 \%$ TWEEN-20) for $30 \mathrm{~min}$ in a humid chamber at $37^{\circ} \mathrm{C}$. Probes were detected using $30 \mu \mathrm{l}$ of 1:10 anti-digoxigenin fluorescein, diluted in detection buffer (4× SSC, $1 \mathrm{mg} \mathrm{ml-1}$ bovine serum albumin and $0.1 \%$ TWEEN-20) for $60 \mathrm{~min}$ in a humid chamber at $37^{\circ} \mathrm{C}$.

Slides were washed three times for $2 \mathrm{~min}$ in $4 \times$ SSC and $0.1 \%$ TWEEN-20. For subsequent immunofluorescence, $50 \mu \mathrm{l}$ of primary antibody against $\gamma \mathrm{H} 2 \mathrm{AFX}$ (Upstate, 16 193), diluted 1:100 in $4 \times$ SSC and 0.1\% TWEEN-20, was added to slides and incubated for $30 \mathrm{~min}$ in a humid chamber at room temperature. Slides were washed for $2 \mathrm{~min}$ in $4 \times \mathrm{SSC}$ and $0.1 \%$ TWEEN-20. Next, $50 \mu \mathrm{l}$ of secondary antibody (AlexaFluor 594 conjugated), diluted 1:100 in $4 \times \mathrm{SSC}$ and $0.1 \%$ TWEEN-20, was added to slides and incubated for $30 \mathrm{~min}$ in a humid chamber at room temperature. Finally, slides were washed for $2 \mathrm{~min}$ in $4 \times \mathrm{SSC}$ and $0.1 \%$ TWEEN-20 and mounted in Vectashield with DAPI.
For RNA FISH analyses, cells were first categorised based upon the presence or absence of a $\gamma \mathrm{H} 2 \mathrm{AFX}$ domain or HORMAD2. The cells were then classified based upon the presence or absence of RNA FISH signals.

\section{Imaging}

Imaging was performed using an Olympus IX70 inverted microscope with a 100-W mercury arc lamp. For chromosome spread and RNA FISH imaging, an Olympus UPlanApo $100 \times / 1.35$ NA oil immersion objective was used. For ovary section imaging, an Olympus UPlanApo 20×/0.75 NA objective was used. A Deltavision RT computer-assisted Photometrics CoolsnapHQ CCD camera with an ICX285 Progressive scan CCD image sensor was utilised for image capture. 16-bit $(1024 \times 1024$ pixels $)$ raw images of each channel were captured and later processed using Fiji.

Acknowledgments We thank Victor Tybulewicz for providing Tc1 mice, Grzegorz Polikiewicz for the assistance with genotyping, Paul Burgoyne for the maintenance of the Tc1 mouse line, NIMR Biological Services for animal husbandry and technical support. This work was supported by the Francis Crick Institute, which receives its core funding from Cancer Research UK, the UK Medical Research Council (U117588498) and the Wellcome Trust. Additional funding was provided by the Deutsche Forschungsgemeinschaft (TO 421/3-1, 421/3-2, 421/4-1 and 421/5-1).

Compliance with ethical standards All animal procedures were in accordance with the United Kingdom Animal Scientific Procedures Act 1986 and were subject to local ethical review.

Conflicts of interest The authors declare that they have no competing interests.

Open Access This article is distributed under the terms of the Creative Commons Attribution 4.0 International License (http:// creativecommons.org/licenses/by/4.0/), which permits unrestricted use, distribution, and reproduction in any medium, provided you give appropriate credit to the original author(s) and the source, provide a link to the Creative Commons license, and indicate if changes were made.

\section{References}

Arnold AP, Chen X, Itoh Y (2012) What a difference an X or Y makes: sex chromosomes, gene dose, and epigenetics in sexual differentiation. In: Handbook of experimental pharmacology., pp 67-88

Baarends WM, Wassenaar E, van der Laan R, Hoogerbrugge J, SleddensLinkels E, Hoeijmakers JH, de Boer P, Grootegoed JA (2005) Silencing of unpaired chromatin and histone $\mathrm{H} 2 \mathrm{~A}$ ubiquitination in mammalian meiosis. Mol Cell Biol 25:1041-1053

Becherel OJ, Yeo AJ, Stellati A, Heng EY, Luff J, Suraweera AM, Woods R, Fleming J, Carrie D, McKinney K, Xu X, Deng C, Lavin MF (2013) Senataxin plays an essential role with DNA damage response proteins in meiotic recombination and gene silencing. PLoS Genet 9:e1003435 
Burgoyne PS, Baker TG (1985) Perinatal oocyte loss in XO mice and its implications for the aetiology of gonadal dysgenesis in XO women. J Reprod Fertil 75:633-645

Burgoyne PS, Sutcliffe MJ, Mahadevaiah SK (1992) The role of unpaired sex chromosomes in spermatogenic failure. Andrologia 24:17-20

Burgoyne PS, Mahadevaiah SK, Turner JM (2009) The consequences of asynapsis for mammalian meiosis. Nat Rev Genet 10:207-216

Celeste A, Petersen S, Romanienko PJ, Fernandez-Capetillo O, Chen HT, Sedelnikova OA, Reina-San-Martin B, Coppola V, Meffre E, Difilippantonio MJ, Redon C, Pilch DR, Olaru A, Eckhaus M, Camerini-Otero RD, Tessarollo L, Livak F, Manova K, Bonner WM, Nussenzweig MC, Nussenzweig A (2002) Genomic instability in mice lacking histone H2AX. Science 296:922-927

Cloutier JM, Mahadevaiah SK, ElInati E, Nussenzweig A, Toth A, Turner JM (2015) Histone H2AFX links meiotic chromosome asynapsis to prophase I oocyte loss in mammals. PLoS Genet 11:e1005462

Daniel K, Lange J, Hached K, Fu J, Anastassiadis K, Roig I, Cooke HJ, Stewart AF, Wassmann K, Jasin M, Keeney S, Toth A (2011) Meiotic homologue alignment and its quality surveillance are controlled by mouse HORMAD1. Nat Cell Biol 13:599-610

Eicher EM, Hale DW, Hunt PA, Lee BK, Tucker PK, King TR, Eppig JT, Washburn LL (1991) The mouse $\mathrm{Y}^{*}$ chromosome involves a complex rearrangement, including interstitial positioning of the pseudoautosomal region. Cytogenet Cell Genet 57:221-230

Fukuda T, Daniel K, Wojtasz L, Toth A, Hoog C (2010) A novel mammalian HORMA domain-containing protein, HORMAD1, preferentially associates with unsynapsed meiotic chromosomes. Exp Cell Res 316:158-171

Garcia-Cruz R, Roig I, Robles P, Scherthan H, Garcia Caldes M (2009) ATR, BRCA1 and gammaH2AX localize to unsynapsed chromosomes at the pachytene stage in human oocytes. Reprod Biomed Online 18:37-44

Handel MA, Schimenti JC (2010) Genetics of mammalian meiosis: regulation, dynamics and impact on fertility. Nat Rev Genet 11: 124-136

Hunt PA, Hassold TJ (2002) Sex matters in meiosis. Science 296: 2181-2183

Ichijima Y, Ichijima M, Lou Z, Nussenzweig A, Camerini-Otero RD, Chen J, Andreassen PR, Namekawa SH (2011) MDC1 directs chromosome-wide silencing of the sex chromosomes in male germ cells. Genes Dev 25:959-971

Kolas NK, Yuan L, Hoog C, Heng HH, Marcon E, Moens PB (2004) Male mouse meiotic chromosome cores deficient in structural proteins SYCP3 and SYCP2 align by homology but fail to synapse and have possible impaired specificity of chromatin loop attachment. Cytogenet Genome Res 105:182-188

Kouznetsova A, Lister L, Nordenskjold M, Herbert M, Hoog C (2007) Bi-orientation of achiasmatic chromosomes in meiosis I oocytes contributes to aneuploidy in mice. Nat Genet 39:966-968

Kouznetsova A, Wang H, Bellani M, Camerini-Otero RD, Jessberger R, Hoog C (2009) BRCA1-mediated chromatin silencing is limited to oocytes with a small number of asynapsed chromosomes. J Cell Sci 122:2446-2452

LeMaire-Adkins R, Radke K, Hunt PA (1997) Lack of checkpoint control at the metaphase/anaphase transition: a mechanism of meiotic nondisjunction in mammalian females. J Cell Biol 139:1611-1619

Luoh SW, Bain PA, Polakiewicz RD, Goodheart ML, Gardner H, Jaenisch R, Page DC (1997) Zfx mutation results in small animal size and reduced germ cell number in male and female mice. Development 124:2275-2284

Mahadevaiah SK, Odorisio T, Elliott DJ, Rattigan A, Szot M, Laval SH, Washburn LL, McCarrey JR, Cattanach BM, Lovell-Badge R, Burgoyne PS (1998) Mouse homologues of the human AZF candidate gene RBM are expressed in spermatogonia and spermatids, and map to a $\mathrm{Y}$ chromosome deletion interval associated with a high incidence of sperm abnormalities. Hum Mol Genet 7:715-727

Mahadevaiah SK, Bourc'his D, de Rooij DG, Bestor TH, Turner JM, Burgoyne PS (2008) Extensive meiotic asynapsis in mice antagonises meiotic silencing of unsynapsed chromatin and consequently disrupts meiotic sex chromosome inactivation. J Cell Biol 182:263-276

Mahadevaiah SK, Costa Y, Turner JM (2009) Using RNA FISH to study gene expression during mammalian meiosis. Methods Mol Biol 558:433-444

Manterola M, Page J, Vasco C, Berrios S, Parra MT, Viera A, Rufas JS, Zuccotti M, Garagna S, Fernandez-Donoso R (2009) A high incidence of meiotic silencing of unsynapsed chromatin is not associated with substantial pachytene loss in heterozygous male mice carrying multiple simple robertsonian translocations. PLoS Genet 5: e1000625

McKee BD, Handel MA (1993) Sex chromosomes, recombination, and chromatin conformation. Chromosoma 102:71-80

Modzelewski AJ, Holmes RJ, Hilz S, Grimson A, Cohen PE (2012) AGO4 regulates entry into meiosis and influences silencing of sex chromosomes in the male mouse germline. Dev Cell 23:251-264

Morelli MA, Cohen PE (2005) Not all germ cells are created equal: aspects of sexual dimorphism in mammalian meiosis. Reproduction 130:761-781

Nagaoka SI, Hodges CA, Albertini DF, Hunt PA (2011) Oocyte-specific differences in cell-cycle control create an innate susceptibility to meiotic errors. Curr Biol 21:651-657

Nagaoka SI, Hassold TJ, Hunt PA (2012) Human aneuploidy: mechanisms and new insights into an age-old problem. Nat Rev Genet 13: 493-504

O'Doherty A, Ruf S, Mulligan C, Hildreth V, Errington ML, Cooke S, Sesay A, Modino S, Vanes L, Hernandez D, Linehan JM, Sharpe PT, Brandner S, Bliss TV, Henderson DJ, Nizetic D, Tybulewicz VL, Fisher EM (2005) An aneuploid mouse strain carrying human chromosome 21 with Down syndrome phenotypes. Science 309:2033-2037

Rogers RS, Inselman A, Handel MA, Matunis MJ (2004) SUMO modified proteins localize to the XY body of pachytene spermatocytes. Chromosoma 113:233-243

Royo H, Polikiewicz G, Mahadevaiah SK, Prosser H, Mitchell M, Bradley A, de Rooij DG, Burgoyne PS, Turner JM (2010) Evidence that meiotic sex chromosome inactivation is essential for male fertility. Curr Biol 20:2117-2123

Royo H, Prosser H, Ruzankina Y, Mahadevaiah SK, Cloutier JM, Baumann M, Fukuda T, Hoog C, Toth A, de Rooij DG, Bradley A, Brown EJ, Turner JM (2013) ATR acts stage specifically to regulate multiple aspects of mammalian meiotic silencing. Genes Dev 27:1484-1494

Shin YH, Choi Y, Erdin SU, Yatsenko SA, Kloc M, Yang F, Wang PJ, Meistrich ML, Rajkovic A (2010) Hormad1 mutation disrupts synaptonemal complex formation, recombination, and chromosome segregation in mammalian meiosis. PLoS Genet 6:e1001190

Taketo T, Naumova AK (2013) Oocyte heterogeneity with respect to the meiotic silencing of unsynapsed $\mathrm{X}$ chromosomes in the $\mathrm{XY}$ female mouse. Chromosoma 122:337-349

Turner JM, Aprelikova O, Xu X, Wang R, Kim S, Chandramouli GV, Barrett JC, Burgoyne PS, Deng CX (2004) BRCA1, histone H2AX phosphorylation, and male meiotic sex chromosome inactivation. Curr Biol 14:2135-2142

Turner JM, Mahadevaiah SK, Fernandez-Capetillo O, Nussenzweig A, Xu X, Deng CX, Burgoyne PS (2005) Silencing of unsynapsed meiotic chromosomes in the mouse. Nat Genet 37:41-47

Vernet N, Mahadevaiah SK, Ojarikre OA, Longepied G, Prosser HM, Bradley A, Mitchell MJ, Burgoyne PS (2011) The Yencoded gene zfy 2 acts to remove cells with unpaired 
chromosomes at the first meiotic metaphase in male mice. Curr Biol 21:787-793

Vigodner M, Morris PL (2005) Testicular expression of small ubiquitin-related modifier-1 (SUMO-1) supports multiple roles in spermatogenesis: silencing of sex chromosomes in spermatocytes, spermatid microtubule nucleation, and nuclear reshaping. Dev Biol 282:480-492

Wojtasz L, Daniel K, Roig I, Bolcun-Filas E, Xu H, Boonsanay V, Eckmann CR, Cooke HJ, Jasin M, Keeney S, McKay MJ, Toth A
(2009) Mouse HORMAD1 and HORMAD2, two conserved meiotic chromosomal proteins, are depleted from synapsed chromosome axes with the help of TRIP13 AAA-ATPase. PLoS Genet 5:e1000702

Wojtasz L, Cloutier JM, Baumann M, Daniel K, Varga J, Fu J, Anastassiadis K, Stewart AF, Remenyi A, Turner JM, Toth A (2012) Meiotic DNA double-strand breaks and chromosome asynapsis in mice are monitored by distinct HORMAD2-independent and -dependent mechanisms. Genes Dev 26:958-973 